\title{
Velocity dependence of the interocular transfer of dynamic motion aftereffects
}

\author{
Ran Tao \\ Department of Ophthalmology, Baogang Hospital, Baotou, Inner Mongolia, People's Republic of China \\ Martin J M Lankheet, Wim A van de Grind $\uparrow$, Richard J A van Wezel \\ Department of Functional Neurobiology and Helmholtz School, Utrecht University, Padualaan 8, \\ NL 3584 CH Utrecht, The Netherlands; e-mail: w.a.vandegrind@bio.uu.nl \\ Received 21 August 2002, in revised form 20 February 2003
}

\begin{abstract}
It is well established that motion aftereffects (MAEs) can show interocular transfer (IOT); that is, motion adaptation in one eye can give a MAE in the other eye. Different quantification methods and different test stimuli have been shown to give different IOT magnitudes, varying from no to almost full IOT. In this study, we examine to what extent IOT of the dynamic MAE (dMAE), that is the MAE seen with a dynamic noise test pattern, varies with velocity of the adaptation stimulus. We measured strength of dMAE by a nulling method. The aftereffect induced by adaptation to a moving random-pixel array was compensated (nulled), during a brief dynamic test period, by the same kind of motion stimulus of variable luminance signal-to-noise ratio (LSNR). The LSNR nulling value was determined in a Quest-staircase procedure. We found that velocity has a strong effect on the magnitude of IOT for the dMAE. For increasing speeds from $1.5 \mathrm{deg} \mathrm{s}^{-1}$ to $24 \mathrm{deg} \mathrm{s}^{-1}$ average IOT values increased about linearly from $18 \%$ to $63 \%$ or from $32 \%$ to $83 \%$, depending on IOT definition. The finding that dMAEs transfer to an increasing extent as speed increases, suggests that binocular cells play a more dominant role at higher speeds.
\end{abstract}

\section{Introduction}

If we look at a well-chosen test stimulus after adapting for some time to visual motion, we usually experience a motion aftereffect (MAE), that is an illusory motion in a direction opposite to the adaptation direction (reviewed by Wade 1994; Wade and Verstraten 1998). A MAE seen on a static test pattern, which is the classical testing situation (Purkinje 1820; Addams 1835; Aristotle 1955) is nowadays called a static MAE or sMAE. Such an SMAE is somewhat paradoxical in the sense that the observer can see motion and is at the same time aware of the fact that neither the test pattern as a whole, nor its details are changing their position in space. One interpretation is that the brain is taking nonconcordant signals from a position analysis system and from an independent motion analysis system into account in the resulting perceptual experience. It has been known since Dvorak (1870) and Wohlgemuth (1911) that the sMAE shows interocular transfer (IOT). That is, adaptation in one eye produces aftereffects measured with the other (unadapted) eye. The current view is that IOT is based on adaptation of binocular neurons that can be stimulated from either eye and tested through the other eye. A review on IOT of MAEs, mostly the sMAE, has been presented by Wade et al (1993). According to this review, IOT of the sMAE for translational motion can, depending on adaptation and testing conditions, vary from $0 \%$ to $78 \%$ and is on average around $50 \%$.

More recently, Hiris and Blake (1992; see also Blake and Hiris 1993) described a dynamic MAE, or dMAE, evoked on a dynamic-noise test pattern after adaptation to moving random-dot patterns. A dMAE lacks the mentioned paradoxical quality of an sMAE and rather looks like real motion. With moving random-pixel arrays (RPAs) or moving Julesz patterns (Julesz 1971) for adaptation, that is random-dot patterns 
where the dots are contiguous square pattern elements, it was subsequently shown that the speed-tunings of sMAE and dMAE differ substantially (Verstraten et al 1998). Whereas the sMAE stops at adaptation speeds above about $20 \mathrm{deg} \mathrm{s}^{-1}$ in the fovea, the dMAE reaches its optimum near the cut-off speed of the sMAE. Conversely the dMAE is very weak or cannot be evoked at all (depending on subjects) for speeds below about $0.5-1 \mathrm{deg} \mathrm{s}^{-1}$, where the sMAE is still at about half its maximum strength (van de Grind et al 2001, their figure 2). Apparently, a population of adapting medium-to-high-speed sensors is responsible for the dMAE, and a population of adapting low-to-medium-speed sensors for the sMAE. Evidence that these populations are independent will be discussed below. We will refer to these hypothetical populations as the high-speed system and the low-speed system, respectively, although it should be kept in mind that the speed-tunings overlap substantially.

In support of this two-system interpretation, an experiment in which subjects adapt to transparently presented opponent motions of high and low speeds shows that one gets a MAE of the low-speed adaptation for a static test and of the high-speed adaptation for a dynamic test (Verstraten et al 1998; see also Verstraten et al 1999). In fact, one can get both MAEs simultaneously, in transparent fashion, by using a test stimulus with both dynamic and static contributions (van der Smagt et al 1999a). This was interpreted to mean that the two MAEs result from independent systems that can each evoke their own percept and do not mutually (intersystemically) interfere. Van de Grind et al (2001) then reported that these low-speed and high-speed systems do not show intersystemic binocular rivalry. If two low-speed (or two high-speed) stimuli of opponent direction are dichoptically presented each to a different eye, one gets (intrasystemic) binocular rivalry. That is, you sometimes see one, then the other, pattern dominate and sometimes you see some form of scrambled mixture of the two. However, if you view a low-speed stimulus with one eye and a high-speed stimulus of opponent direction with the other, you don't get rivalry, but a transparent motion percept. It appears that the two systems even have their own rivalry stages.

Most work on MAEs has been done with moving extended-contour stimuli, prototypical examples of which are sine-wave patterns, plaids, and line patterns. If tested with a static test pattern, these stimuli generate sMAEs, that is MAEs with an upper adaptation speed limit on the order of $20 \mathrm{deg} \mathrm{s}^{-1}$ (van de Grind et al 2001, their figure 2). In 1983 Green et al discovered that one can extend the upper speed limit of MAEs by testing with a homogenously flickering field. They called this the flicker MAE, which we will abbreviate as fMAE. The fMAE is promoted by low spatial frequency, high contrast, and high temporal frequency of the adaptation stimulus (Green et al 1983). In other words, minimising position information (the number of contours) in the adaptation stimulus, and maximising high-frequency flicker strengthens the fMAE. The fMAE shows no interocular transfer (Green et al 1983). This fact and its higher speed range makes it likely that the fMAE is based on a different process from that responsible for the sMAE.

For the same class of adaptation stimuli (moving sine-wave patterns) it was later found that a counterphase flickering sine-wave test pattern, can also give a strong MAE (eg von Grünau 1986; Ashida and Osaka 1994), which we will call the cMAE (c for 'counterphase' testing). The cMAE shows strong IOT whereas the fMAE shows none. As will be discussed below, the cMAE has properties different from those of the dMAE, sMAE, and fMAE. This means that we now have four types of MAE:

(1) The sMAE requires low-speed motion adaptation, and a static test pattern. It has a somewhat paradoxical appearance of motion as well as a fixed position, and shows on average an IOT of around 50\% (Wade et al 1993). It can be evoked for almost any moving pattern (also random-dot patterns and RPAs) 
and is strongest if the static test pattern has spatial properties similar to those of the moving pattern (Thompson 1998).

(2) The fMAE requires adaptation to moving coarse high-contrast patterns, and homogeneous flicker as a test pattern. It shows no IOT and can be evoked for higher adaptation speeds than the sMAE. Otherwise not much is known about this MAE.

(3) The cMAE occurs after adaptation to moving spatial patterns if the test stimulus is a counterphase flickering version of the adaptation pattern. It is different from the previous two MAEs in that it can show up to $100 \%$ IOT. Moreover, the cMAE is speed-tuned (Ashida and Osaka 1995), whereas the sMAE has been shown to be temporal-frequency-tuned (Pantle 1974; Ashida and Osaka 1995). More differences between the sMAE and cMAE have been reported (eg Ashida and Osaka 1994; Nishida et al 1994; Nishida and Sato 1995) so it is necessary to discriminate between the two. Nishida et al (1994) found $100 \%$ IOT of the cMAE as far as MAE duration is concerned, and partial transfer if measured with a nulling technique (Nishida and Ashida 2000). For a very specific case of a mixture of first-order motion (our present topic) and second-order motion (outside the scope of this paper), Nishida and Ashida (2001) even report more than 100\% IOT. We will briefly return to this finding in section 4. Von Grünau (2002) recently studied the cMAE after adaptation to moving plaids with a variable amount of coherence between the two grating components of the plaids. His results suggest that the cMAE for plaids is based on activation both of low-level neurons (eg in V1) and higherlevel pattern-units (eg in V2 or MT). Like Nishida et al (1994), he found a virtually complete IOT for cMAE durations. (Von Grünau called the 'cMAE' a 'dynamic MAE').

(4) The dMAE requires adaptation to moving random textures, random dots, or RPAs, and testing with nondrifting dynamic noise. As reviewed above, it has an optimum at higher speeds than the sMAE and an upper cut-off speed on the order of $100 \mathrm{deg} \mathrm{s}^{-1}$ (van de Grind et al 2001, their figure 2). Because the dMAE has several properties in common with the cMAE, the question arises whether the cMAE and dMAE are really different MAEs or rather the same effect, but measured with different stimuli. Van der Smagt (1999, see also van der Smagt et al 2000) studied the speed dependence of MAE durations for adaptation to moving RPAs using three different test stimuli: a static test to get an sMAE, a dynamic noise test to measure the dMAE, and a counterphase flickering RPA to get a cMAE. He found that the SMAE and dMAE are reasonably pure representations of adaptational gain changes in a low-speed system and a highspeed system, respectively, whereas the cMAE for RPAs had mixed properties. For the time being, it therefore seems necessary to assume that the cMAE and dMAE have partly or completely different origins. What then is known about IOT of dMAEs?

Raymond (1993) used random-dot kinematograms as adaptation stimuli, and measured IOT of a coherent motion-detection threshold-elevation caused by such an adaptation. As her threshold measurements involved the use of random-dot kinematograms, the aftereffect in question must have been related to the dMAE. At a fixed speed of $1.83 \mathrm{deg} \mathrm{s}^{-1}$, she measured threshold elevation both in the adapted and in the contralateral eye. It showed an almost complete IOT, suggesting that neurons that were adapted most and tested best by random-dot kinematograms, are all binocular. McColl and Mitchell (1998) also found an almost complete IOT of threshold-elevation for 
coherent motion detection after monocular adaptation to moving random-dot patterns. Unfortunately, it is hard to relate these threshold elevations quantitatively to conventional measures of IOT, such as the ratio of MAE durations (or perceived speeds) in an adapted and nonadapted eye. In fact, a model analysis by van de Grind et al (2003) suggests that there is a free parameter involved in comparing threshold elevation values to MAE values such as nulling or duration results. Steiner et al (1994), who used a nulling method to quantify the strength of the dMAE, reported an IOT of $70 \%-83 \%$ for a translational dMAE. (For rotation and expansion motion they found higher percentages, but we will concentrate exclusively on translation.)

A study on binocular MAEs for adaptation to random-dot kinematograms and testing with dynamic noise was reported by Grunewald and Mingolla (1998). It is important in the present context, as our study was partly motivated by the same theoretical model (Grunewald and Lankheet 1996; van de Grind et al 2003). They worked with bivectorial adaptation regimes in which the left eye viewed motion in the $60^{\circ}$ direction, the right eye in the $150^{\circ}$ direction (the direction towards 3 o'clock being called zero). They stimulated either the left-eye alone or the right-eye alone or both eyes together during adaptation and test. (Not all possible combinations were used.) In the case of binocular adaptation and binocular testing both the adaptation and the test showed binocular rivalry. The direction of monocular MAEs was not influenced by contralateral adaptation of the perpendicular direction; however, on binocular adaptation and testing, the dMAE was vertically downward, so opposite to the vector sum of the adaptation velocities. Thus, there is a binocular integration stage combining adaptation directions in the dMAE, but this shows up only if the test is binocular, not if it is monocular. In their second experiment Grunewald and Mingolla (1998) specifically studied IOT for the same adaptation conditions. The transferred dMAEs tended to have the same direction as the ipsilateral dMAE, so IOT was direction specific. Unfortunately, they did not measure the strength of IOT. Yet their findings provide important boundary conditions for extension of our MAE models. One difference with other studies of the dMAE, as reviewed above, was the speed. Grunewald and Mingolla (1998) used a relatively high adaptation speed $\left(20 \mathrm{deg} \mathrm{s} \mathrm{s}^{-1}\right)$, whereas the other studies were done with low speeds $\left(1.5-2 \mathrm{deg} \mathrm{s}^{-1}\right)$.

In surveying the literature on IOT of the dMAE we noticed that the influence of speed has been neglected. Yet, work in our own laboratory (eg Verstraten et al 1998, 1999; van der Smagt et al 1999a, 1999b) shows that speed strongly affects MAEs and binocular interactions (van de Grind et al 2001). We therefore decided to quantify the influence of speed on IOT of the dMAE. Hiris and Blake (1992), Blake and Hiris (1993), Raymond (1993), McColl and Mitchell (1998), Castet et al (2002) all used rather low speeds $\left(1.5-2 \mathrm{deg} \mathrm{s}^{-1}\right)$. The study of Verstraten et al (1998) showed that the dMAE is short lasting at such low speeds and is much stronger at higher speeds, say above $4-8 \mathrm{deg} \mathrm{s}^{-1}$. It is therefore of great theoretical importance to measure IOT of the dMAE as a function of speed. That is the purpose of this study.

We decided to use a nulling technique, because it is more straightforward to relate nulling values to adaptation strength (gain) than detection threshold-elevations (van de Grind et al 2003). Unlike Hiris and Blake (1992), Blake and Hiris (1993), Raymond (1993), Steiner et al (1994), McColl and Mitchell (1998) and others, we will not use sparse random-dot kinematograms, but dense moving random textures (or RPAs). The reasons why this is a better choice have recently been discussed and illustrated extensively by Castet et al (2002). They showed that the MAE strength is underestimated when measured by nulling with a low-density random-dot pattern. Our use of moving RPAs and nulling with the luminance signal-to-noise ratio (LSNR), see below, circumvents these problems. Another disadvantage of the stimuli used so far to study IOT of dMAEs is their low temporal frequency. Frame rates were mostly in the order of 
$10-15 \mathrm{~s}^{-1}$, but it has been shown (eg van der Smagt et al 1999a) that the high-speed dMAE first starts to dominate perception for frame rates above $20-25 \mathrm{~s}^{-1}$. Therefore we will use a higher frame rate in this study $\left(50 \mathrm{~s}^{-1}\right)$.

\section{Methods}

\subsection{Stimulus generation}

Moving RPAs of $256 \times 256$ pixels ranging in speed from $0.75 \mathrm{deg} \mathrm{s}^{-1}$ to $24 \mathrm{deg} \mathrm{s}^{-1}$ were generated by a custom-built hardware device, controlled by a Macintosh computer. The patterns were presented on a CRT monitor (Electrohome model EVM-1200, $\mathrm{P} 4$ phosphor) at a frame rate of $50 \mathrm{~s}^{-1}$. The viewing distance was $2 \mathrm{~m}$, resulting in a pixel size of $1 \mathrm{~min}$ of arc, which means that a speed of 1 pixel per frame corresponds to $V=1.5 \mathrm{deg} \mathrm{s}$. The average luminance of all RPAs was $50 \mathrm{~cd} \mathrm{~m}^{-2}$. We used the LSNR method developed by van Doorn and Koenderink (1982a, 1982b). On every frame of the 'signal' RPA, a new noise RPA is added pixel-by-pixel in such a way that the rms (root-mean-square) contrast of the sum $C=\left(C_{\mathrm{s}}^{2}+C_{\mathrm{n}}^{2}\right)^{1 / 2}$ is kept constant. $C_{\mathrm{s}}$ is the rms contrast of the coherently moving signal RPA and $C_{\mathrm{n}}$ of the added dynamic noise patterns. The ratio of signal pattern to noise-pattern rms-contrast is changed according to the responses of the subject. It is the LSNR value $S=C_{\mathrm{s}}^{2} / C_{\mathrm{n}}^{2}$. The luminances of noise and signal pixels were controlled by 10-bit digital-to-analog converters. Both monitors were controlled by the same special-purpose hardware (Noise Image Machine, NIM), which allows synchronisation down to the single-pixel level. In the present experiments, this high synchronisation resolution was less important, as we only stimulated one eye at a time with motion or noise. The other eye then viewed a gray screen of equal average luminance. According to Lehmkuhle and Fox (1976), this gives better IOT than occlusion of the nonstimulated eye. In a pilot experiment with optical shutters we confirmed their findings. The use of an equal luminance gray field prevents strong switching transients, which - as in the fMAEappear to prevent or weaken IOT.

\subsection{Adaptation and nulling paradigm}

The adaptation stimulus was a moving RPA at a maximum LSNR value (which was 400 in our setup), and ranging in speed from $0.75 \mathrm{deg} \mathrm{s}^{-1}$ to $24 \mathrm{deg} \mathrm{s}^{-1}$. The nulling stimulus had the same spatiotemporal properties as the adaptation stimulus, and differed only in LSNR value. Both adaptation and nulling stimuli had a constant rms contrast of $0.7(70 \%)$.

\subsection{Direction discrimination threshold measurements}

To measure the (unadapted) direction-discrimination threshold $S_{\mathrm{d}}$ we used a Queststaircase procedure (Watson and Pelli 1983) with a two-alternative forced-choice method. The stimulus moved randomly to the left or right, and it was the subject's task to indicate the direction of motion. The Quest procedure lowers the LSNR for correct responses and increases the LSNR for wrong answers in such a way that the staircase converges on the $80 \%$ point. The staircase consisted of 50 trials. Presentation duration was $0.5 \mathrm{~s}$ for these trials, and the subject initiated the next trial with a start button.

\section{4 dMAE nulling measurements}

After a pre-adaptation of $15 \mathrm{~s}$, a periodic sequence started of $5 \mathrm{~s}$ top-up adaptation and $0.5 \mathrm{~s}$ testing (nulling). During test intervals the subject had to indicate the perceived direction of motion in a two-alternative forced-choice task. If the adaptation direction is to the right and the MAE therefore to the left, the nulling stimulus during test intervals also moves to the right. If the MAE is stronger than the nulling stimulus, motion to the left is perceived; if the nulling stimulus is stronger than the MAE, motion to the right is seen. A Quest-staircase procedure was used to estimate the 
$50 \%$ point in 50 test intervals. The LSNR of this $50 \%$ point is the nulling value $S_{0}$, at the chosen speed.

\subsection{Observers, viewing, and presentation conditions}

Three observers with normal or corrected-to-normal visual acuity participated in the experiment. Two of the observers (CM, SK) were naïve as to the purposes of the experiment. Each eye viewed a separate monitor through an adjustable stereoscopic mirror arrangement. The room was dimly lit, so that the contours of the monitors and other equipment were visible. The head was stabilised in space with a chin-and-forehead rest. The two monitors were adjusted and calibrated to be virtually identical at the beginning of the experiments. Preceding every measurement session, motion discrimination thresholds were measured for the whole range of speeds, with each monitor used in turn to check that they were still identical. No significant changes in these thresholds were found during the project (however, see the results). The test stimulus was monocularly presented either to the adapted eye or to the unadapted eye (IOT). There were in all four conditions: adapt left eye and test left eye (LL), adapt right eye and test right eye (RR), adapt right eye and test left eye (RL), adapt left eye and test right eye (LR). A gray screen with the same mean luminance was presented to the nonadapting or to the non-tested eye. In order to avoid accumulation of adaptation, the direction of the adapting stimulus was always opposite to that of the previous and next staircase and the sequence of the four conditions (LL, RR, LR, RL) was selected randomly.

\section{Results}

The main results, as presented in figure 1, show that nulling values both in adapted and in contralateral eyes increase with increasing adaptation speed. Nulling values in the contralateral eye were always lower than in the ipsilateral (the adapted) eye, so IOT was never complete for the range of speeds used in this experiment.

The results in figure 1 show a consistent increase in nulling values as velocity increases. Although there are some differences between observers, the similarities are much more striking. In all cases, results for the contralateral eye (open triangles) are close to the detection thresholds (closed circles) at low speeds, rise more steeply than the ipsilateral nulling values (open squares) for increasing speeds, and approach the latter at higher speeds. This is precisely the pattern one would expect for an increase of IOT with speed, whatever the precise choice of an IOT quantification (see below).

Rather than performing an eye-dominance test with some nonrelated stimulus, we decided to measure transfer both from left to right and from right to left for each observer, as shown in the two columns in figure 1. On the whole, the results for the two eyes of each of the three observers are qualitatively highly similar. Yet we were surprised to see that the nulling values measured in the right eye were consistently higher for all three subjects than in their left eyes. At first sight this suggests a difference between the two monitors. However, as described in the methods section, they were calibrated to be equal, and we compared the two monitors at the start of each session by measuring direction-discrimination thresholds separately for each of the two monitors. This procedure never showed any difference at all. In fact, figure 1 underscores that the direction-discrimination thresholds of the two eyes (and thus monitors) were never significantly different. The eye-differences show up only for the nulling values. We have no clear explanation for this phenomenon, but fortunately it can not in any way influence the conclusions regarding IOT. Relations between the ipsilateral and contralateral nulling values are highly similar in the two eyes. This justifies averaging across eyes for each of the subjects. IOT quantification will then be based upon these averaged data. 

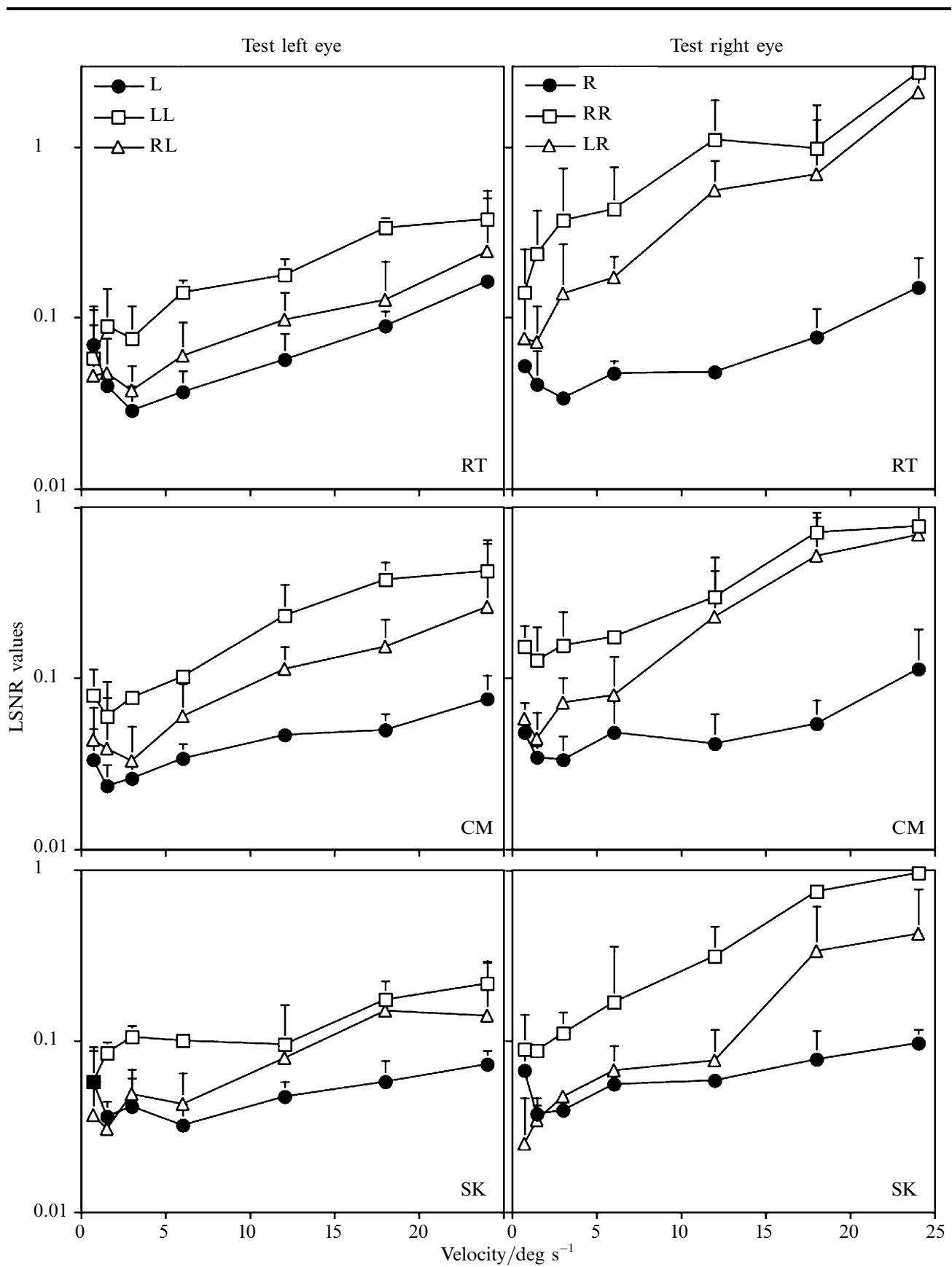

Figure 1. MAE nulling values measured by testing the left eye (left column) or testing the right eye (right column) for adaptation of the same eye (open squares) or of the other eye (open triangles). $\mathrm{L}$ stands for left eye and $\mathrm{R}$ for right eye, the first letter in combinations gives the adapted eye, the second letter the tested eye. Thus, LL and RR refer to nulling measurements in the adapted eye, whereas LR and RL refer to nulling values measured in the nonadapted eye. These nulling values are presented as a function of speed. We used speeds of $0.5,1,2,4,8,12$, and 16 pixels per frame, where 1 pixel per frame equals $1.5 \mathrm{deg} \mathrm{s}^{-1}$. Direction-discrimination thresholds for the left eye (L) and right eye (R) are presented for comparison (closed circles). At low speeds, data for RL and LR are close to the threshold, indicating a very small IOT. For higher speeds, they approach the values of LL and RR, respectively, indicating a high IOT. 
In many studies in the literature IOT is simply quantified as the ratio between contralateral and ipsilateral MAE strength, where 'strength' is represented by MAE duration or nulling values. In our view this is not a correct quantification, because it does not take any baseline threshold value into account. The strength of adaptation is proportional to how much a nulling value is suprathreshold. Therefore either the difference or the ratio between $S_{0}$ and $S_{\mathrm{d}}$ is a better measure of adaptation strength. If we choose the difference we get:

$$
\mathrm{IOT}=100 \frac{S_{0 \mathrm{c}}-S_{\mathrm{d}}}{S_{0 \mathrm{i}}-S_{\mathrm{d}}} \%
$$

where $S_{0 \mathrm{c}}$ and $S_{o \mathrm{i}}$ are the contralateral and ipsilateral nulling values, respectively, and $S_{\mathrm{d}}$ is the direction-discrimination threshold, which is about equal in the two eyes. We cannot just choose a ratio rather than a difference as measure of adaptation strength, because then $S_{\mathrm{d}}$ would disappear from the IOT formula. However, we can take a log ratio as a measure of adaptation strength, to obtain the following alternative IOT formula, in which the influence of $S_{\mathrm{d}}$ as baseline is preserved:

$$
\mathrm{IOT}=100 \frac{\log S_{0 \mathrm{c}}-\log S_{\mathrm{d}}}{\log S_{0 \mathrm{i}}-\log S_{\mathrm{d}}} \% .
$$

This formula represents a ratio of linearly measured vertical distances between pairs of curves in figure 1, which are almost straight lines in the presented log-linear plots. Of course, $S_{\mathrm{d}}$ values depend on the chosen threshold criterion (in our case $80 \%$ correct), but this is a relatively minor factor. It does not influence the change of IOT with speed, only the vertical position of the curves in figure 2, and only slightly.

Formulae (1) and (2) were used to calculate IOT values from the data in figure 1. The results are presented in figure 2. In these calculations, results for the two eyes were first averaged per observer (LL combined with RR, and LR combined with RL). Then we calculated IOT values with formulae (1) and (2) for each of the observers separately. Finally we determined the average across observers, leaving out the data for the lowest speed $\left(0.75 \mathrm{deg} \mathrm{s}^{-1}\right)$. At the lowest speed, some observers showed lower nulling values than discrimination thresholds, making these data unusable. At $1.5 \mathrm{deg} \mathrm{s}^{-1}$, only the results for SK could not be used for this same reason, so we used the average of two observers for this speed. For all other speeds, the average is based on the data of all three observers. In general, observer SK has lower IOT values than the other two observers.

Figure 2 shows that the average IOT is quite modest at low speeds, especially if formula (1) is used, and increases with increasing speed. The average across observers can be described quite well by the following linear regression lines (dashed lines in figure 2):

$$
\begin{array}{ll}
\text { formula (1) gives } & \text { IOT }=14.74+2.03 V\left(r^{2}=0.99\right) \\
\text { formula (2) gives } & \mathrm{IOT}=28.42+2.29 V\left(r^{2}=0.97\right)
\end{array}
$$

If we suppose that these regression lines can be extrapolated to higher speeds than used by us, this would mean a full IOT (100\%) around $42 \mathrm{deg} \mathrm{s}^{-1}$ or $31 \mathrm{deg} \mathrm{s}^{-1}$ for formulae (1) and (2), respectively. We also included data points for the individual observers in figure 2 as open circles for formula (1) and open squares for formula (2). Observer SK always has the lowest IOT values. Results for the other two observers are not only higher, but generally also quite close together. Seemingly missing open symbols fall under the closed symbols. 


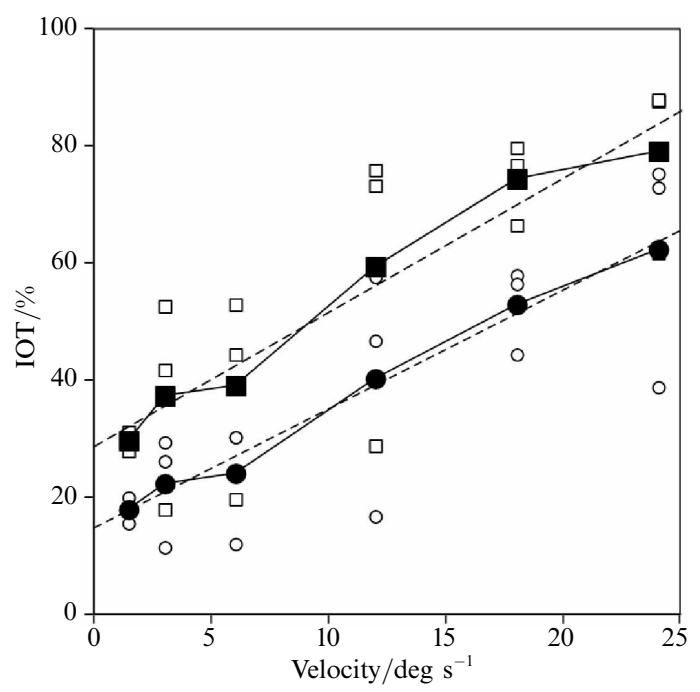

Figure 2. Overall average magnitude of IOT as a function of adaptation velocity. Two different formulae are used to quantify IOT, as explained in the text. Small open circles are calculated from formula (1) of the text, for each of the three observers, by averaging left to right and right to left IOT per observer. Small open squares give results for the same observers calculated from formula (2). The larger filled symbols resulted from averaging IOT values across subjects according to formula (1) (filled circles) and formula (2) (filled squares). Dashed linear-regression lines [formulae (3) and (4) of the text] describe the averaged results quite well.

\section{Discussion}

Previous studies, as reviewed in section 1, unanimously reported that IOT of the sMAE is partial (Wade et al 1993), that IOT for the cMAE is stronger and can be complete for MAE durations (eg Nishida and Ashida 2000; von Grünau 2002), even though it is smaller for nulling thresholds (Nishida and Ashida 2000). For the dMAE, there are reports of almost $100 \%$ IOT of a motion-detection threshold-elevation due to adaptation (Raymond 1993; McColl and Mitchell 1998). However, all studies with a nulling technique report a less than complete IOT for the dMAE (Hiris and Blake 1992; Blake and Hiris 1993; Steiner et al 1994; this study). The reasons for this discrepancy are not fully clear at this time, but in agreement with Steiner et al (1994) we think the explanation is of a methodological nature. It is not a priori clear that and how a detection threshold elevation relates to MAE strength. Some cells might increase their threshold during adaptation without contributing to a dMAE, for example because they are not sensitive to the MAE test stimuli. Such cells would contribute to the threshold-elevation effect even though they are irrelevant to the MAE. Until these threshold-elevation measures are experimentally or theoretically related to those of MAE strength, we think it is preferable to use direct MAE measures, like nulling values. Of course, MAE duration measures can also be used, even though they tend to be noisier than nulling values (van de Grind et al 2003).

In an appendix to their paper van der Smagt et al (1999b) compared IOT for the dMAE and SMAE on the basis of MAE durations. Although it was not mentioned in their paper, they used diagonal motion with a speed of $32 \mathrm{deg} \mathrm{s}^{-1}$ for the dMAE and of $1 \mathrm{deg} \mathrm{s}^{-1}$ for the sMAE (personal communication). Their choice of different speeds resulted in quite similar IOT percentages of about $60 \%-70 \%$ for the two types of MAE. However, van der Smagt et al (1999b) did not take any baseline threshold into account. For our present nulling thresholds this would mean a definition such as IOT $=100 S_{0 \mathrm{c}} / S_{0 \mathrm{i}}$, and for our subjects a regression line describing these IOT values 
for the dMAE as a function of speed is IOT $=37.7+1.2 \mathrm{~V}\left(r^{2}=0.99\right)$. This gives an IOT value of $66 \%$ at our highest speed of $24 \mathrm{deg} \mathrm{s}^{-1}$ and predicts (by linear extrapolation) an IOT value of around $76 \%$ at $32 \mathrm{deg} \mathrm{s}^{-1}$. These figures are not unlike the results reported by van der Smagt et al (1999b), but the comparison is less straightforward than it seems, despite the use of similar stimuli in the two studies. The main problem is that there is a nonlinear relation between dMAE duration and $S_{0}$ values, that involves some parameters of the underlying gain-control system (van de Grind et al 2003). Such parameters have not been determined in the present study nor in the one by van der Smagt et al (1999b). More in general we think it is important in quantifying IOT to be aware of the different definitions used by different authors, and the problems of relating IOT values determined from MAE durations, nulling values, and threshold changes. The only sensible way to compare such different quantification methods is through a common model.

One simple explanation of the present findings is that monocular motion sensors of equal velocity-specificity map onto binocular units for the same velocity via a fixed coupling factor, and that this factor is higher for channels tuned to higher speeds. With automatic gain controls of the type described by van de Grind et al (2003) both in the monocular and in the binocular channels we can indeed reproduce the results of the present study. Consequently, a two-level model suffices, with a monocular gain control at one stage and a binocular gain control at the next stage. In this context, it is interesting to note that such a simple two-stage model can even produce an IOT of more than $100 \%$ for high coupling factors. This is a consequence of the fact that test signals pass through a lowered gain via the adapted eye, but not via the contralateral side. Thus in contralateral testing a test signal arriving at the input of the binocular cell can be stronger than for ipsilateral testing. Under appropriate circumstances this leads to a more than $100 \%$ transfer of MAE strength, at least in our simulations. Nishida and Ashida (2001) reported a MAE for contralateral testing that indeed exceeded the effect for ipsilateral testing, but in their case it concerned second-order motion, on which our experiments and models have nothing to say.

Electrophysiological studies have shown that cells in extra-striate visual areas of macaque, eg the middle temporal (MT) area, are uniformly binocular, whereas cells in striate area V1 vary in their degree of binocularity. Therefore, a two-stage substrate, as required by our above explanation, is in principle available in the primate visual system. What is more, van Wezel and Britten (2002) have recently shown a strong correlation between adaptation of MT-cells in a nonhuman primate and the psychophysical effects of the same type of motion adaptation in human observers. We think it would be premature to stress analogies between models and electrophysiology much further at this point. The first task ahead would rather seem to be the development of a full-blown model explaining both the direction-integration results of Grunewald and Mingolla (1998), and our present results on speed-dependence of IOT. It would also be interesting to explore IOT at still higher speeds to see whether the trend towards higher IOT for high adaptation speeds continues up to full transfer or even beyond.

\section{Conclusions}

We measured the IOT magnitude of dMAEs for a range of velocities with a nulling method. We found that IOT increases with increasing speed, although it never reaches $100 \%$ in the speed range we used (up to $24 \mathrm{deg} \mathrm{s}^{-1}$ ). The interocular transfer for medium velocities resembles that of the sMAE, while the transfer for high velocities is similar to that found for the cMAE with nulling methods. The findings suggest that the mapping gains from monocular onto binocular cells increase with the speed to which motion channels are tuned. 
Acknowledgments. We thank Frans Verstraten, Ans Koenderink-van Doorn, and Maarten van der Smagt for their constructive comments on earlier versions of the manuscript.

\section{References}

Addams R, 1835 "Optische Täuschung nach Betrachtung eines in Bewegung begriffenen Körpers" Annalen der Physik und Chemie $\mathbf{3 4} 348$

Aristotle 1955 Parva Naturalia Revised text with introduction and commentary by W D Ross (Oxford, UK: Oxford University Press)

Ashida H, Osaka N, 1994 "Difference of spatial frequency selectivity between static and flicker motion aftereffects" Perception 231313 - 1320

Ashida H, Osaka N, 1995 "Motion aftereffect with flickering test stimuli depends on adapting velocity" Vision Research 351825 - 1833

Blake R, Hiris E, 1993 "Another means for measuring the motion aftereffect" Vision Research $331589-1592$

Castet E, Keeble D R T, Verstraten F A J, 2002 "Nulling the motion aftereffect with dynamic random-dot stimuli: Limitations and implications" Journal of Vision $2302-311$

Doorn A J van, Koenderink J J, 1982a "Temporal properties of the visual detectability of moving spatial white noise" Experimental Brain Research 45 179-188

Doorn A J van, Koenderink J J, 1982b "Spatial properties of the visual detectability of moving spatial white noise" Experimental Brain Research 45 189-195

Dvorak V, 1870 "Versuche über die Nachbilder von Reizveränderungen" Sitzungsberichte der Wiener Akademie der Wissenschaften $\mathbf{6 1} 257-262$

Green M, Chilcoat M, Stromeyer III C F, 1983 "Rapid motion aftereffect seen within uniform flickering test fields" Nature $30461-62$

Grind W A van de, Hof P van, Smagt M J van der, Verstraten F A J, 2001 "Slow and fast visual motion channels have independent binocular rivalry stages" Proceedings of the Royal Society of London, Series B $\mathbf{2 6 8} 437-443$

Grind W A van de, Lankheet M J M, Tao R, 2003 "A gain-control model relating nulling results to the duration of dynamic motion aftereffects" Vision Research $43117-133$

Grünau M von, 1986 "A motion aftereffect for long-range stroboscopic apparent motion" Perception \& Psychophysics $\mathbf{4 0} 31-38$

Grünau M von, 2002 "Bivectorial transparent stimuli simultaneously adapt mechanisms at different levels of the motion pathway" Vision Research 42 577-587

Grunewald A, Lankheet M J M, 1996 "Orthogonal motion aftereffect illusion predicted by a model of cortical motion processing" Nature $38358-360$

Grunewald A, Mingolla E, 1998 "Motion after-effect due to binocular sum of adaptation to linear motion" Vision Research $382963-2971$

Hiris E, Blake R, 1992 "Another perspective on the visual motion aftereffect" Proceedings of the National Academy of Sciences of the USA $899025-9028$

Julesz B, 1971 Foundations of Cyclopean Perception (Chicago, IL: University of Chicago Press)

Lehmkuhle S W, Fox R, 1976 "On measuring interocular transfer" Vision Research $16428-430$

McColl L, Mitchell D E, 1998 "Stereodeficient subjects show substantial differences in interocular transfer of two motion adaptation aftereffects" Vision Research $381889-1900$

Nishida S, Ashida H, 2000 "A hierarchical structure of motion system revealed by interocular transfer of flicker motion aftereffects" Vision Research 40 265-278

Nishida S, Ashida H, 2001 "A motion aftereffect seen more strongly by the non-adapted eye: evidence of multistage adaptation in visual motion processing" Vision Research 41561 - 570

Nishida S, Ashida H, Sato T, 1994 "Complete interocular transfer of motion aftereffect with flickering test" Vision Research $342707-2716$

Nishida S, Sato T, 1995 "Motion aftereffect with flickering test patterns reveals higher stages of motion processing" Vision Research 35 477-490

Pantle A, 1974 "Motion aftereffect magnitude as a measure of the spatio-temporal response properties of direction sensitive analyzers" Vision Research $141229-1236$

Purkinje J E, 1820 "Beiträge zur näheren Kenntniss des Schwindels aus heautognostischen Daten" Medizinische Jahrbücher des kaiserlich-königlichen österreichischen Staates 6 79-125

Raymond J E, 1993 "Complete interocular transfer of motion adaptation effects on motion coherence thresholds" Vision Research 33 1865-1870

Smagt M J van der, 1999 Integration and Segregation Mechanisms in Human Motion Vision $\mathrm{PhD}$ thesis, Utrecht University (ISBN 90-393-2100-0), Utrecht, The Netherlands

Smagt M J van der, Verstraten F A J, Grind W A van de, 1999a "New transparent motion aftereffect" Nature Neuroscience 2 595-596 
Smagt M J van der, Verstraten F A J, Grind W A van de, 2000 "Do motion aftereffects depend on test-pattern temporal frequency?" Perception 29 Supplement, 28

Smagt M J van der, Verstraten F A J, Vaessen E B P, Londen T van, Grind W A van de, 1999b "Motion aftereffect of combined first-order and second-order motion" Perception 28 1397-1411

Steiner V, Blake R, Rose D, 1994 "Interocular transfer of expansion, rotation, and translation motion aftereffects" Perception 231197 - 1202

Thompson P, 1998 "Tuning of the motion aftereffect", in The Motion Aftereffect. A Modern Perspective Eds G Mather, F A J Verstraten, S Anstis (Cambridge, MA: MIT Press) pp $41-55$

Verstraten F A J, Smagt M J van der, Fredericksen R E, Grind W A van de, 1999 "Integration after adaptation to transparent motion: static and dynamic test patterns result in different aftereffect directions" Vision Research $39803-810$

Verstraten F A J, Smagt M J van der, Grind W A van de, 1998 "Aftereffect of high-speed motion" Perception $271055-1066$

Wade N J, 1994 "A selective history of the study of the visual motion aftereffects" Perception 23 $1111-1134$

Wade N J, Swanton M T, de Weert C M M, 1993 "On interocular transfer of motion aftereffects" Perception $221365-1380$

Wade N J, Verstraten F A J, 1998 "Introduction and historical overview", in The Motion Aftereffect. A Modern Perspective Eds G Mather, F A J Verstraten, S Anstis (Cambridge, MA: MIT Press) pp $1-23$

Watson A B, Pelli D G, 1983 "Quest: a Bayesian adaptive psychometric method" Perception \& Psychophysics $33113-120$

Wezel R J A van, Britten K H, 2002 "Motion adaptation in area MT" Journal of Neurophysiology $883469-3476$

Wohlgemuth A, 1911 "On the after-effect of seen movement" British Journal of Psychology Monograph Supplement 1 1-117 (whole monograph) 


\section{PERCEPTION}

VOLUME 322003

www.perceptionweb.com

Conditions of use. This article may be downloaded from the Perception website for personal research by members of subscribing organisations. Authors are entitled to distribute their own article (in printed form or by e-mail) to up to 50 people. This PDF may not be placed on any website (or other online distribution system) without permission of the publisher. 